\title{
Searches for New Physics at Tevatron: Most Recent Results
}

\author{
A. V. Popov" \\ (for Collaboration D0) \\ Institute for High Energy Physics, Protvino, Moscow oblast, 142284 Russia \\ Received March 23, 2010; in final form, August 6, 2010
}

\begin{abstract}
The most recent results obtained from searches of particles and phenomena beyond the Standard Model (new physics) in the D0 and CDF experiments at the Tevatron accelerator (FNAL, USA) on the basis of statistics corresponding to the integrated luminosity of up to $4 \mathrm{fb}^{-1}$ in 2009 are considered. Particular attention is given to the most recent results on searches for a supersymmetric Higgs boson.
\end{abstract}

DOI: $10.1134 / \mathrm{S} 1063778811020177$

\section{INTRODUCTION}

Many years of investigations have proven the viability of the Standard Model [1], which describes satisfactorily observed processes and phenomena in particle physics. Yet, it is not free from serious drawbacks that motivate searches for Standard Model extensions that could provide answers to the remaining questions. In particular, the physics behind the hierarchy problem, the dark-matter phenomenon, and unification of gauge couplings calls for explanation.

Until recently, the Tevatron accelerator (FNAL, USA), which is characterized by the c.m. collidingbeam energy of $1.96 \mathrm{TeV}$ and at which a wide range of studies including searches for supersymmetric partners of quarks, gluons, and gauge and Higgs bosons and searches for leptoquarks, extra spatial dimensions and effects associated with them, and new heavy gauge bosons and quarks is being performed, was the appropriate testing ground for new-physics searches.

The most recent results obtained on the basis of statistics corresponding to the integrated luminosity of about $2-4 \mathrm{fb}^{-1}$ are considered in this article (previous review articles of the present author were devoted to results based on smaller statistics-see [2]). Particular attention is given here to describing, on the basis of the minimal supersymmetric model (MSSM) and next-to-MSSM (nMSSM), the most recent results stemming from searches for supersymmetric Higgs bosons.

\section{SEARCHES FOR SUPERSYMMETRIC HIGGS BOSONS}

In MSSM, the Higgs sector is extended in relation to the Standard Model. Specifically, it includes

*E-mail: Alexei.Popov@ihep.ru Operated by Fermi Research Alliance, LLC under Contract No. DE-AC02-07CH11359 with the United States Department of Energy. two Higgs doublets. Of these, one is associated with up-type fermions, while the other is associated with down-type fermions. Accordingly, there are five Higgs bosons - three neutral (scalar $h$ and $H$ and pseudoscalar $A$ ) and two charged $\left(H^{ \pm}\right)$. In the leading-order ( $\mathrm{LO}$ ) approximation, the Higgs sector in MSSM can be described in terms of two parameters. For these, one most frequently takes the Higgs boson mass $\left(M_{A}\right)$ and the ratio of the vacuum expectation values of two Higgs fields $(\tan \beta)$. The region of small $M_{A}$ and large $\tan \beta$ is the most interesting for searches for sypersymmetric Higgs bosons at the Tevatron accelerator, since, in this region, the cross section for the production of neutral supersymmetric Higgs bosons increases by a factor of about $2 \tan ^{2} \beta$. In this region, the main modes of decay of the neutral Higgs boson are $\phi \rightarrow b \bar{b}$ (the branching fraction is about $90 \%$ ) and $\phi \rightarrow \tau \tau$ (the branching fraction is about $10 \%$ ); here, $\phi$ is the generic notation for the neutral Higgs bosons $h, H$, and $A$.

Searches for neutral supersymmetric Higgs bosons at the Tevatron accelerator are performed by using the following signatures: the signature of direct Higgs boson production and its subsequent decay to two $\tau$ leptons (this is a relatively "clean" signature accompanied by a small background of QCD processes, but the respective branching fraction is modest, about $10 \%$ ), the signature of associated Higgs boson and $b$-quark production followed by Higgs boson decay to a $b \bar{b}$ pair (this process has a larger branching ratio of about $90 \%$, but it is more strongly contaminated by the background of QCD processes), and the signature of associated Higgs boson and $b$-quark production followed by Higgs boson decay to a $\tau \tau$ pair (a reduced background of QCD processes and a higher sensitivity in the region of small $M_{A}$ ). 


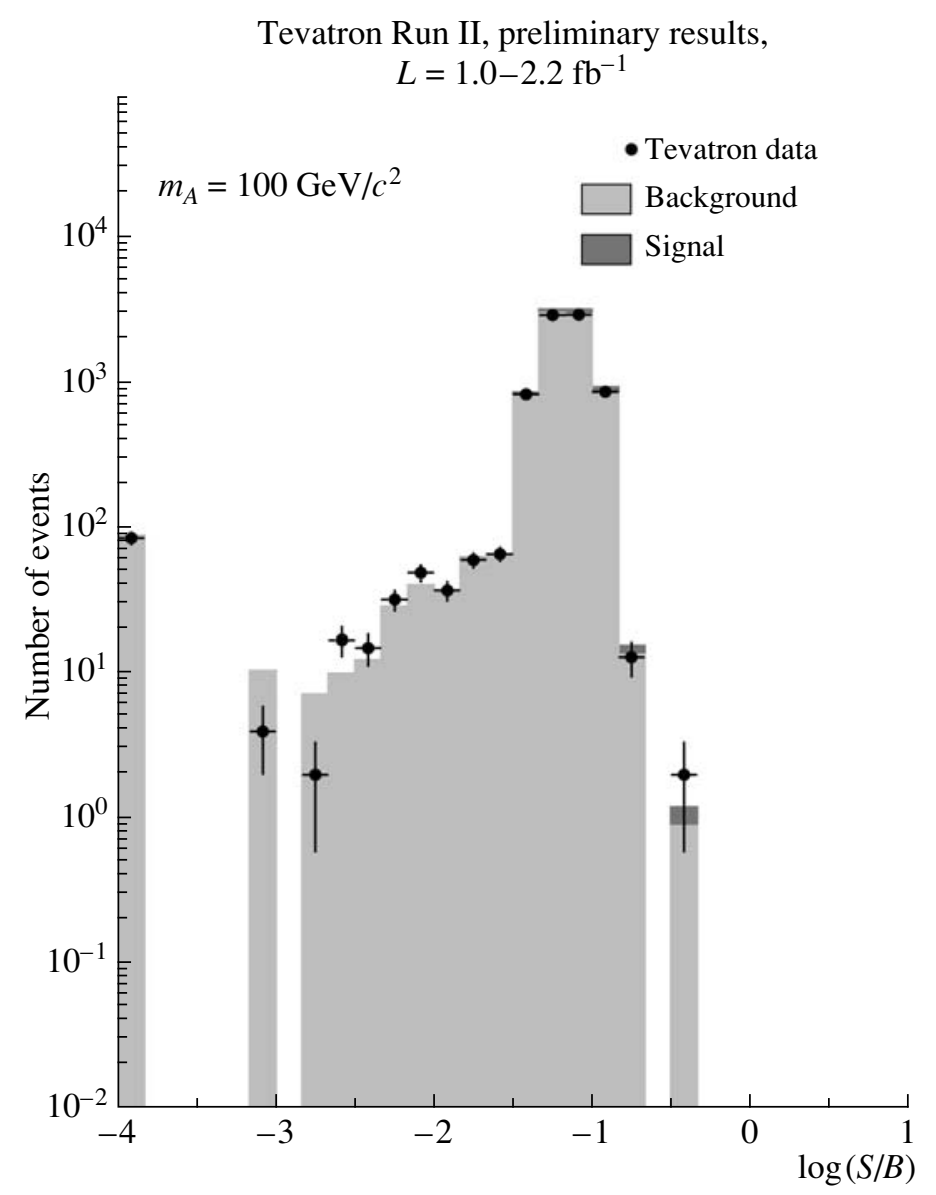

Fig. 1. Distribution of $\log (S / B)$ for data, background, and $\operatorname{signal}\left(M_{A}=100 \mathrm{GeV} / c^{2}\right)$ : analysis of the global statistics of the D0 and CDF experiments.

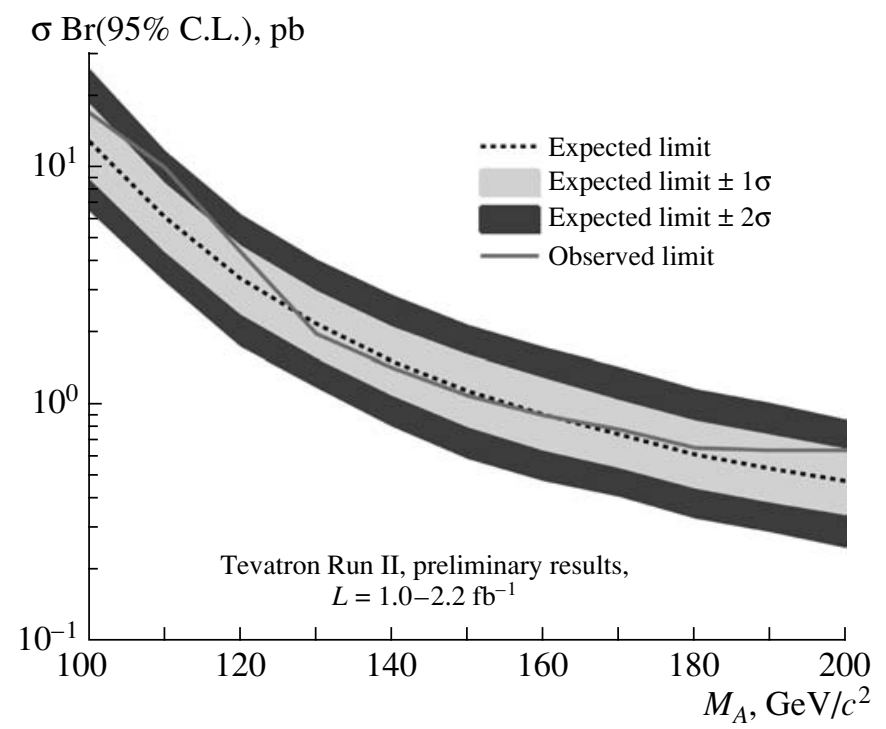

Fig. 2. Limit on the cross section for the production of a neutral supersymmetric Higgs boson versus its mass: analysis of global statistics from the D0 and CDF experiments. 


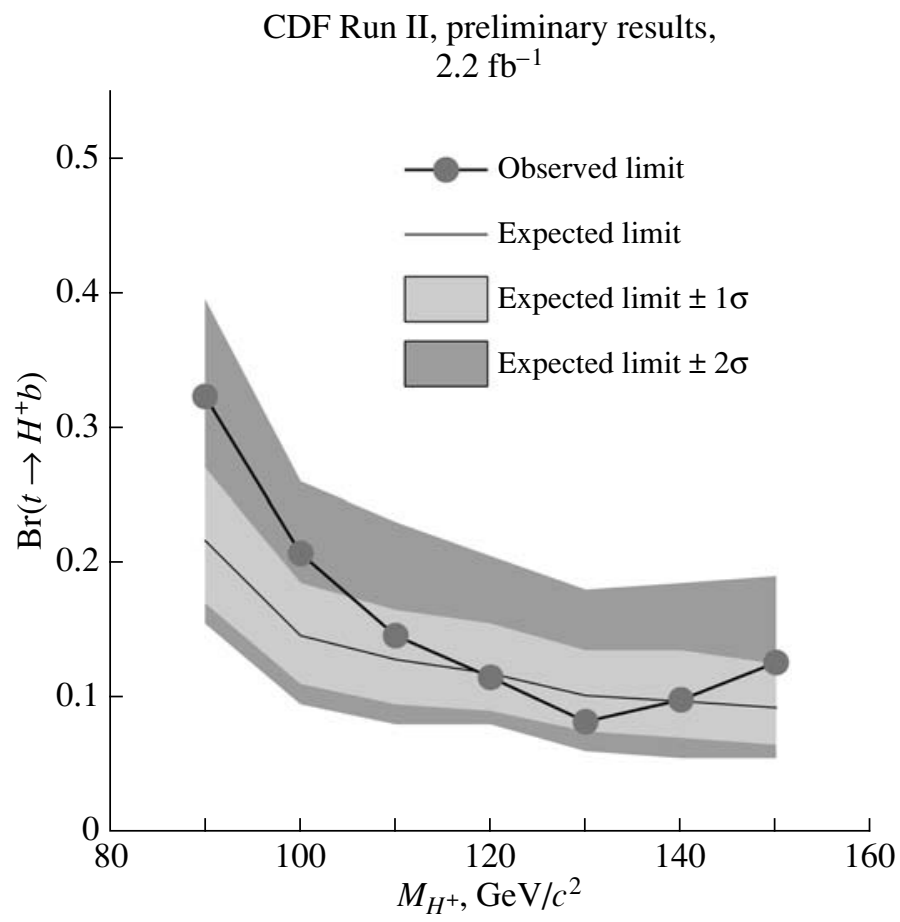

Fig. 3. Limit on $\operatorname{Br}\left(t \rightarrow H^{+} b\right)$ under the assumption of $\operatorname{Br}\left(H^{+} \rightarrow c \bar{s}\right)=1$ versus the $H^{+}$mass.

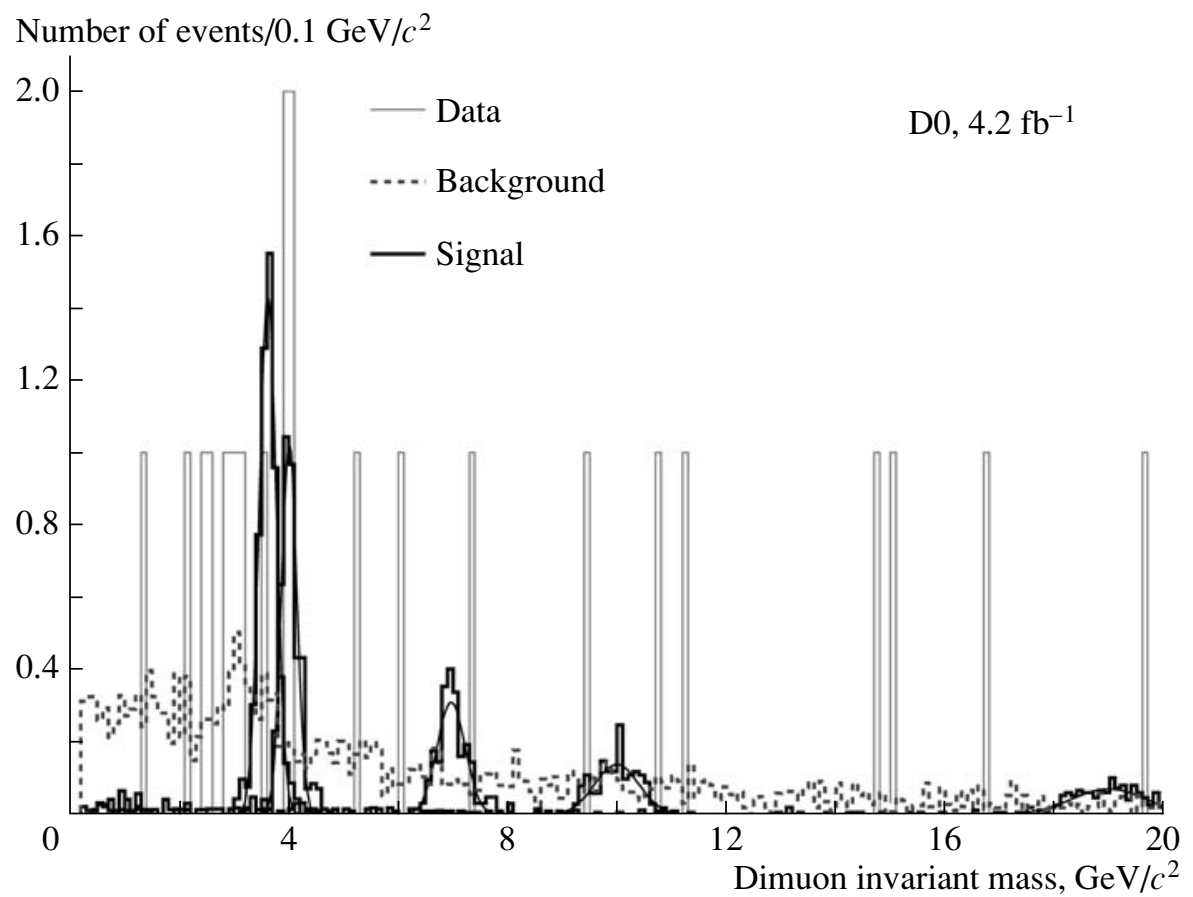

Fig. 4. Invariant-mass distribution of dimuons for events (data, background, and signal) that passed all selections and cuts $\left[M_{a}=3.6,4,7,10\right.$, and $19 \mathrm{GeV} / c^{2} ; \sigma(p \bar{p} \rightarrow h+X)=1.9 \mathrm{pb} ; \operatorname{Br}(h \rightarrow a a)=1 ;$ and $\left.M_{h}=100 \mathrm{GeV} / c^{2}\right]$.

\subsection{Searches for Neutral Higgs Bosons}

A combined result of the D0 and CDF experiments on searches for neutral supersymmetric Higgs bosons on the basis of global statistics corresponding to the integrated luminosity of $1.8 \mathrm{fb}^{-1}(\mathrm{CDF})$ and $2.2 \mathrm{fb}^{-1}$ (D0) was presented in 2009 [3]. The processes in which the direct production of a neutral Higgs boson and its production in association with a $b$ quark are 
followed by Higgs boson decay to a $\tau \tau$ pair were considered. Both the leptonic and hadronic modes of $\tau$ decays were taken into account.

The respective analysis did not reveal any piece of evidence of the production of a neutral supersymmetric Higgs boson. The observed picture is well described by the Standard Model. Figure 1 shows the distribution of $\log (S / B)$, where $S$ is the value expected for the signal at the parameter value of $M_{A}=$ $100 \mathrm{GeV}$, while $B$ is the expected background from Standard Model processes. In Fig. 2, the resulting limit on the cross section for the production of a neutral Higgs boson is shown as a function of its mass.

Also, new limits on the cross section for the production of a neutral sypersymmetric Higgs boson were obtained in the D0 experiment in 2009, both direct and associated neutral Higgs boson production followed by Higgs boson decay to a $\tau \tau$ or a $b \bar{b}$ pair being considered there. The analysis was performed on the basis of statistics corresponding to the integrated luminosity of $2.2(\phi \rightarrow \tau \tau), 1.2$ $(b \phi \rightarrow b \tau \tau)$, and $2.6 \mathrm{fb}^{-1}(b \phi \rightarrow b b \bar{b})$ [4]. This analysis did not reveal significant deviations from the Standard Model predictions. Accordingly, new limits on the cross section for the production of a neutral Higgs boson were established. In the parameter plane spanned by $M_{A}$ and $\tan \beta$, the region up to values of $\tan \beta \approx 30$ or 35 could be excluded in the mass range $120-160 \mathrm{GeV} / c^{2}$.

\subsection{Searches for Charged Higgs Bosons}

The results of the CDF experiment on searches for charged supersymmetric Higgs bosons on the basis of statistics corresponding to the integrated luminosity of $2.2 \mathrm{fb}^{-1}$ were reported in 2009 [5], $t \bar{t}$ pair production followed by $t$-quark decay to $H^{+} b$ being considered there. In MSSM, the branching fraction for the decay process $t \rightarrow H^{+} b$ is relatively large if $\tan \beta$ is small (less than or about unity) or great (greater than or about 15). In the first case and at a low mass of $H^{+}$(not greater than $130 \mathrm{GeV} / c^{2}$ ), the decay $H^{+} \rightarrow c \bar{s}$ is dominant, while, at a larger mass, the decay $\mathrm{H}^{+} \rightarrow t^{*} \bar{b} \rightarrow W b \bar{b}$ comes to the fore. In the second case, the decay $H^{+} \rightarrow \tau^{+} \nu$ prevails.

The $t \rightarrow H^{+} b$ topology, where $H^{+} \rightarrow c \bar{s}$, was studied in the case being considered. The invariantmass distribution of dijets was considered in searches for deviations from Standard Model predictions (the process $t \rightarrow W^{+} b$ followed by the hadronic decay of $W$ is the main background process here). No statistically significant deviations from Standard Model predictions were discovered; accordingly, new limits on $\operatorname{Br}\left(t \rightarrow H^{+} b\right)$ were set. These limits are shown in Fig. 3 under the assumption of $\operatorname{Br}\left(H^{+} \rightarrow c \bar{s}\right)=1$ and versus the mass of the charged Higgs boson.

\subsection{Searches for Neutral Higgs Bosons within nMSSM}

Within nMSSM, the scalar Higgs boson $(h)$ decays in the majority of cases to a pair of light neutral pseudoscalar Higgs bosons $(a)$. In the region $2 m_{\mu}<$ $M_{a} \lesssim 3 m_{\pi}$, boson $a$ decays to a $\mu \mu$ pair in nearly $100 \%$ of cases. As the mass of boson $a$ increases, hadronic decay modes come to be dominant, while, in the mass range $m_{\tau}<M_{a}<2 m_{b}$, the decay mode $a \rightarrow \tau \tau$ prevails.

In 2009, the D0 Collaboration presented the result of searches for a light pseudoscalar Higgs boson in the process $h \rightarrow a a, a a \rightarrow \mu \mu \mu \mu$ or $a a \rightarrow \mu \mu \tau \tau$ on the basis of statistics corresponding to the integrated luminosity of $4.2 \mathrm{fb}^{-1}$ [6]. Events involving either two pairs of collinear (consequence of small $M_{a}$ ) muons or events containing one pair of collinear muons and a large missing energy, as well as one unisolated or weakly isolated muon or electron from tau-lepton decay, were selected. In the case being considered, the background is dominated by QCD processes and the decays $Z / \gamma^{*} \rightarrow \mu \mu+$ jets.

Figure 4 shows the invariant-mass distribution of dimuons for data (after all selections and cuts), the background from Standard Model processes, and the signal for various values of $M_{a}\left(M_{a}=\right.$ $3.6,4,7,10$, and $19 \mathrm{GeV} / c^{2}$ ). In the mass region around $4 \mathrm{GeV} / c^{2}$, there is some excess of the number of events in data above the background ( 4 events in data versus 1.1 background events and 3.3 signal events), but the scarcity of statistics does not yet permit drawing any far-reaching conclusions.

\section{SUPERSYMMETRY}

Supersymmetry (SUSY) theory postulates the presence of symmetry between boson and fermion states and predicts the existence of supersymmetric partners for Standard Model particles. Such partners differ only in spin (bosons correspond to fermions, and vice versa). The concept of $R$ parity, which is a multiplicative quantum number equal to +1 for Standard Model particles and -1 for their supersymmetric partners, is introduced in the theory. In minimal supersymmetric extensions of the Standard Model (Minimal Supersymmetric Standard Model, or MSSM), one postulates $R$-parity conservation (yet, there are supersymmetry models involving $R$-parity violation), which has two important implications. First, supersymmetric particles are produced in pairs. 
Second, the lightest of supersymmetric particles (LSP) must be stable, providing a natural solution to the dark-matter problem. So far, experiments did not reveal any experimental indication of the existence of sypersymmetric particles, and this implies their large masses and, hence, a large mass difference between Standard Model particles and their supersymmetric partners. This in turn means that supersymmetry is a broken symmetry. Several supersymmetry-breaking mechanisms have been proposed; of these, we will be interested first of all in the minimal supergravity (mSUGRA) model, where supersymmetry is broken via interaction with gravity. This model is characterized by five parameters: universal masses for scalar and gauge superpartners at the Grand Unification scale, $m_{0}$ and $m_{1 / 2}$; a universal trilinear coupling constant at the Grand Unification scale, $A_{0}$; the ratio of the vacuum expectation values of the Higgs fields, $\tan \beta$; and the sign of the higgsino mass parameter, $\operatorname{sgn}(\mu)$.

\subsection{Searches for Supersymmetric Partners of thet Quark}

In 2009, the D0 Collaboration presented a refined result on searches for supersymmetric partners of the $t$ quark in the channel $\tilde{t} \bar{t} \rightarrow b b e^{ \pm} \mu^{\mp} \tilde{\nu} \bar{\nu}$. This result was obtained on the basis of statistics corresponding to the integrated luminosity of $3.1 \mathrm{fb}^{-1}$ [7]. In analyzing respective data, it was assumed that the supersymmetric partner of the $t$ quark decays through the channel $\tilde{t} \rightarrow b l \tilde{\nu}$ and that the supersymmetric neutrino (sneutrino) $\tilde{\nu}$ appears as the LSP. Thus, the final state involves two unlikely charged leptons, two jets formed by $b$ quarks, and a large missing energy carried away by the LSP.

Here, the background receives a dominant contribution from processes leading to the production of isolated pairs of electrons and muons: $Z / \gamma^{*} \rightarrow$ $\tau^{+} \tau^{-}, W W, W Z, Z Z$, and $t t$. The PYTHIA generator [8] was used to estimate this background. Events in which electrons, muons, or jets were misidentified or in which the missing energy was measured incorrectly are yet another source of background. The background from such processes was estimated directly on the basis of data.

After all selections and cuts, 288 events were selected in the data, the background from Standard Model processes being estimated at $303 \pm 20$ (stat.) events. Thus, it can be concluded that no manifest indication of the pair production of a supersymmetric partner of the $t$ quark was discovered in the above statistics and within the topology being considered. Limits on the $\tilde{t}$ mass versus the $\tilde{\nu}$ mass at a $95 \%$ C.L. were obtained and presented in Fig. 5. For a large value of the mass difference $m_{\tilde{t}}-m_{\tilde{\nu}}$, the constraint on the mass of the lightest supersymmetric partner of the $t$ quark at a $95 \%$ C.L. is $m_{\tilde{t}}>200 \mathrm{GeV} / c^{2}$.

In 2009, the CDF Collaboration presented results obtained from searches of the supersymmetric partner of the $t$ quark in the channels $\tilde{t} \rightarrow c \tilde{\chi}_{1}^{0}$ and $\tilde{t} \rightarrow$ $b \tilde{\chi}_{1}^{ \pm} \rightarrow b l^{ \pm} \nu_{l} \tilde{\chi}_{1}^{0}$ on the basis of statistics corresponding to the integrated luminosity of, respectively, 2.6 and $2.7 \mathrm{fb}^{-1}$ [9]. For the signatures in those searches, use was made of events involving two jets from heavy quarks and a large missing energy in the first case and events involving two jets from heavy quarks, two leptons, and a large missing energy in the second case.

After all selections and cuts, 115 and 57 events in the data were selected for the channels $\tilde{t} \rightarrow c \tilde{\chi}_{1}^{0}$ and $\tilde{t} \rightarrow b l^{ \pm} \nu_{l} \tilde{\chi}_{1}^{0}$, respectively, the expected background level being $132.0 \pm 24.4$ events for the former and $56.4 \pm 7.2$ events for the latter. Obviously, the available data are well described within the Standard Model; therefore, new limits on the cross sections for the production of $\tilde{\tilde{t}}$ pairs were set. In the $m_{\tilde{t}}-m_{\tilde{\chi}_{1}^{0}}$ mass plane, Fig. 6 shows the region excluded by a data analysis for the channel $\tilde{t} \rightarrow c \tilde{\chi}_{1}^{0}$.

\subsection{Searches for Supersymmetric Partners of Gauge Bosons}

Supersymmetric partners of gauge bosons were sought in channels involving three leptons and a large missing energy in the final state. The most probable processes featuring supersymmetric particles and leading to this topology at hadron colliders are processes involving the double production of charged $\left(\tilde{\chi}_{1}^{ \pm}\right)$and neutral $\left(\tilde{\chi}_{2}^{0}\right)$ superpartners thereupon decaying to leptons and LSP particles through intermediate gauge bosons and sleptons $\left(p \bar{p} \rightarrow \tilde{\chi}_{1}^{ \pm} \tilde{\chi}_{2}^{0} \rightarrow\right.$ $\left.l l l \nu \tilde{\chi}_{1}^{0} \tilde{\chi}_{1}^{0}\right)$. In order to improve the detection efficiency for such processes, particle identification is usually required only for two leptons-that is, final states featuring $e e, \mu \mu, e \mu$ plus an isolated track in the central tracking system and a large missing energy are considered.

In 2009, the CDF Collaboration presented a new combined result for all of the topologies considered above [10]. The total statistical sample for analysis corresponded to the integrated luminosity of $3.2 \mathrm{fb}^{-1}$. After all selections and cuts, the number of selected events (summed over all final states being considered) was 7 for the data and $10.84 \pm 1.34$ for Standard Model backgrounds-that is, no excess of events in the data over the expected number of events from Standard Model background processes was discovered. A new upper limit was set on the cross section 


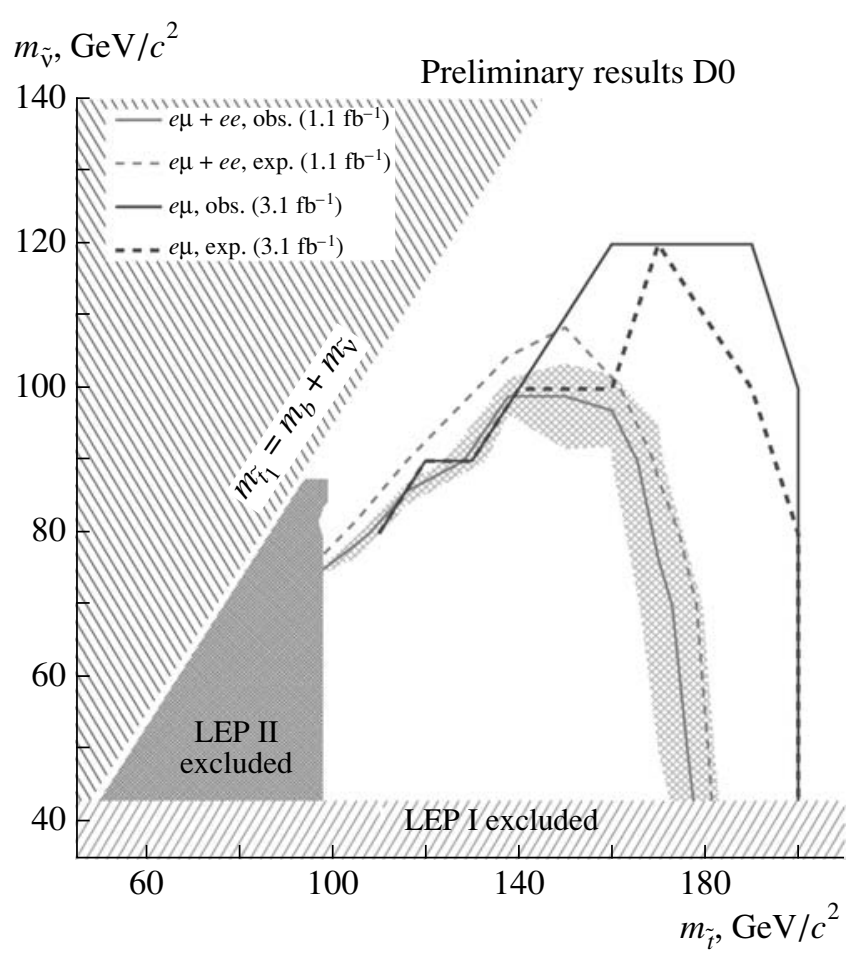

Fig. 5. Region excluded in the $\left(m_{\tilde{t}}, m_{\tilde{\nu}}\right)$ mass plane upon a data analysis as contrasted against the result obtained previously in the D0 experiment on the basis of statistics corresponding to the integrated luminosity of $1.1 \mathrm{fb}^{-1}$ and against the respective results obtained at the LEP collider.

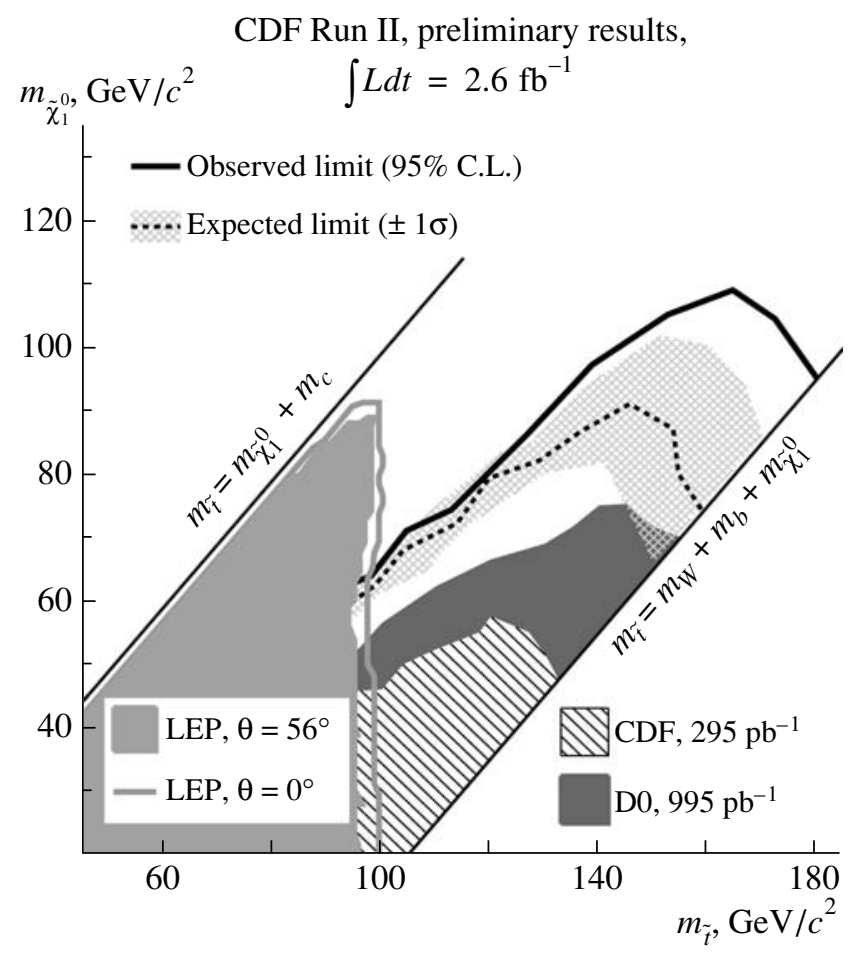

Fig. 6. Region excluded in the $m_{\tilde{t}}-m_{\tilde{\chi}_{1}^{0}}$ mass plane (at a $95 \%$ C.L.) upon the respective data analysis $\left(\tilde{t} \rightarrow c \tilde{\chi}_{1}^{0}\right.$ channel) as contrasted against the results of earlier experiments. 


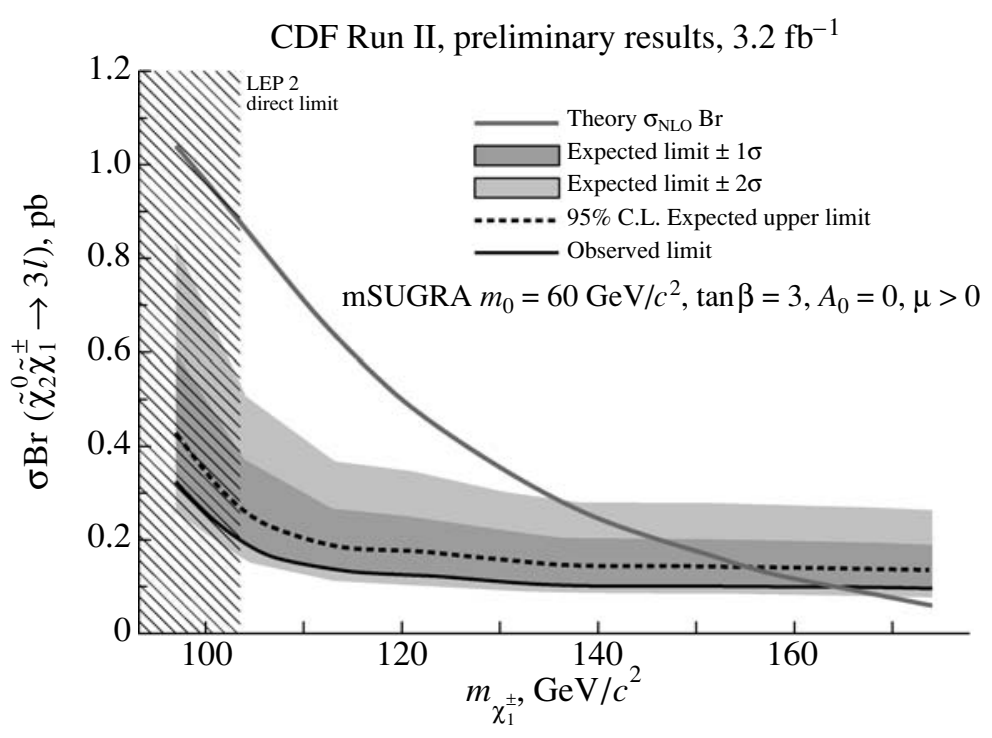

Fig. 7. Upper limit on $\sigma\left(\tilde{\chi}_{1}^{ \pm} \tilde{\chi}_{2}^{0}\right) \operatorname{Br}(3 l)$ as a function of the $\tilde{\chi}_{1}^{ \pm}$mass along with the theoretical value of the cross section for the process under study in the next-to-leading (NLO) approximation.

$\sigma\left(p \bar{p} \rightarrow \tilde{\chi}_{1}^{ \pm} \tilde{\chi}_{2}^{0}\right) \operatorname{Br}\left(\tilde{\chi}_{1}^{ \pm} \tilde{\chi}_{2}^{0} \rightarrow 3 l\right)$ versus the $\tilde{\chi}_{1}^{ \pm}$mass (Fig. 7), and a new lower limit of $164 \mathrm{GeV} / c^{2}$ on the $\tilde{\chi}_{1}^{ \pm}$mass was obtained (for the mSUGRA model parameters set to $m_{0}=60 \mathrm{GeV} / c^{2}, A_{0}=0, \tan \beta=$ 3 , and $\mu>0$ ).

\section{SEARCHES FOR LEPTOQUARKS}

The existence of leptoquarks, which are scalar and vector bosons having a fractional electric charge (an integral multiple of $1 / 3$ ), carrying both lepton and quark quantum numbers, and accordingly decaying to a lepton and quark, are predicted in many Standard Model extensions, such as Grand Unified Theories, technicolor, and various theories assuming the composite structure of quarks. Within such theories, it is additionally assumed that leptoquarks may decay only to leptons and quarks of respective generations; therefore, one distinguishes between first-, second-, and third-generation leptoquarks. Double production is the main process leading to leptoquark production at hadron colliders. By the way, the cross section for leptoquark production depends on the leptoquark mass, but it does not depend on leptoquark coupling to the corresponding quark or lepton. If we denote by $\beta=\operatorname{Br}(L Q \rightarrow l q)$ the branching fraction for the decay process $L Q \rightarrow l q$, then $\operatorname{Br}(L Q \rightarrow \nu q)=1-\beta$ and $\operatorname{Br}(L Q \overline{L Q} \rightarrow l \nu q q)=2 \beta(1-\beta)$.

In 2009, the D0 Collaboration presented the result obtained from searches for third-generation leptoquarks in $p \bar{p} \rightarrow L Q_{3} L Q_{3} \rightarrow \nu_{\tau} \nu_{\tau} b b$ topology on the basis of statistics corresponding to the integrated luminosity of $4.0 \mathrm{fb}^{-1}[11]$. Two jets identified as jets from $b$ quarks and a large missing energy served as a signature for these searches. It should be noted that, apart from leptoquark searches, this signature may be used to seek the lightest supersymmetric partner of the $b$ quark decaying through the channel $\tilde{b}_{1} \rightarrow b \tilde{\chi}_{1}^{0}$.

After the application of all selections and cuts, three candidates were selected versus the expected background of $3.2 \pm 0.3$ (stat.) \pm 0.6 (syst.) events from Standard Model processes. Obviously, these data are described satisfactorily on the basis of the Standard Model; accordingly, a new limit was set on the cross section for the double production of thirdgeneration leptoquarks. The respective lower limit on the leptoquark mass was $252 \mathrm{GeV} / c^{2}$ at a $95 \%$ C.L. for $\beta=0$. Also, new limits on the cross section for the production of the lightest supesymmetric partner of the $b$ quark were also obtained from that analysis.

\section{SEARCHES FOR HEAVY RESONANCES DECAYING TO AN ELECTRON-POSITRON PAIR}

The Standard Model gauge group $S U(3)_{C} \otimes$ $S U(2)_{L} \otimes U(1)_{L}$ may be part of a larger gauge group-for example, $S O(10)$ or $E(6)$ [12]. This assumption is used to construct various models within Grand Unified Theories (GUTs). In many GUT models featuring spontaneous symmetry breaking, the $U(1)$ group survives at relatively low energies, leading to the existence of heavy neutral gauge bosons, which are frequently denoted by $Z^{\prime}$. Such $Z^{\prime}$ bosons are coupled to fermions in the Standard Model through electroweak interactions. At hadron 
Table 1. Observed lower limits on the $Z^{\prime}$ mass within various scenarios of the model based on the $E(6)$ gauge group and on the graviton mass within the Randall-Sundrum (RS) model at various values of model parameters at a 95\% C.L.

\begin{tabular}{c|c|c|c|c|c|c|c|c|c}
\hline Model & $Z_{\mathrm{SSM}}^{\prime}$ & $Z_{\psi}^{\prime}$ & $Z_{\chi}^{\prime}$ & $Z_{\eta}^{\prime}$ & $Z_{I}^{\prime}$ & $Z_{s q}^{\prime}$ & $Z_{N}^{\prime}$ & $G\left(k / M_{\mathrm{Pl}}=0.1\right)$ & $G\left(k / M_{\mathrm{Pl}}=0.07\right)$ \\
\hline$M_{Z^{\prime}, G}\left[\mathrm{GeV} / c^{2}\right]$ & 950 & 763 & 800 & 810 & 692 & 719 & 744 & 786 & 708 \\
\hline
\end{tabular}

Table 2. Heavy-resonance $\left(Z^{\prime}, W^{\prime}\right.$, and $\left.G\right)$ mass regions (in $\mathrm{GeV} / c^{2}$ units) excluded at a $95 \%$ C.L.

\begin{tabular}{c|c|c}
\hline$Z^{\prime}$ & $W^{\prime}$ & $G$ \\
\hline $247-545$ & $284-515$ & $<606$ \\
\hline
\end{tabular}

colliders, such particles can be found, for example, in the processes $p \bar{p} \rightarrow Z^{\prime} \rightarrow e e$.

Such particles appear not only in GUTs but also in models involving extra spatial dimensions. For example, the Randall-Sundrum (RS) model [13] predicts the presence of heavy gravitons, which may decay to a ee pair. The graviton mass $M_{G}$ and the dimensionless coupling $k / M_{\mathrm{Pl}}$ to Standard Model fields, where $k^{2}$ characterizes the spacetime curvature of the extra dimension, are basic parameters of the model.

In 2009, the D0 Collaboration presented results obtained from searches for similar heavy neutral resonances decaying to an electron-positron pair. These searches were based on statistics corresponding to the integrated luminosity of $3.6 \mathrm{fb}^{-1}$ [14]. It is noteworthy that a similar analysis performed earlier in the CDF experiment on the basis of statistics corresponding to the integrated luminosity of $2.5 \mathrm{fb}^{-1}$ [15] revealed some excess of events in data over the expected number of events from Standard Model background processes in the region of dielectron invariant masses around $240 \mathrm{GeV} / c^{2}$. The respective results of the D0 experiment do not confirm this observationthe data fit well in Standard Model predictions, showing no excess of events. New upper limits on the cross sections for $Z^{\prime}$ and $G$ production were deduced within the corresponding models, and new lower limits on the masses of these particles were obtained and are presented in Table 1.

\section{SEARCHES FOR HEAVY RESONANCES DECAYING TO $W^{+} W^{-}$AND $W^{ \pm} Z$}

In 2009, the CDF Collaboration presented the results of an analysis devoted to searches for heavy resonances decaying to $W^{+} W^{-}$or $W^{ \pm} Z^{0}$ pairs [16]. A final state featuring an electron, two jets, and a large missing mass were considered in this analysisthat is, events where the first $W$ boson decays to an electron and a neutrino and where the second ( $W$ or $Z$ ) boson decays through a hadronic channel via the formation of two jets were selected. The total statistical sample used in that analysis corresponds to the integrated luminosity of $2.9 \mathrm{fb}^{-1}$. The results obtained in this way were interpreted within the hypotheses of the existence of heavy $Z^{\prime}$ and $W^{\prime}$ bosons, as well as within the RS model, which involves a heavy graviton $\left(k / M_{\mathrm{Pl}}=0.1\right)$.

In order to isolate a signal in each of the models being considered, use was made of various cuts on the total transverse energy of $e \nu$ and jet $_{1} j_{\text {jet }}$ pairs. As a result, 75 events against the expected background of $75.62 \pm 2.48$ (stat.) \pm 11.23 (syst.) events from Standard Model processes were selected for the hypothesis of a heavy graviton. For the hypothesis of heavy- $Z^{\prime}$ production, 51 events were selected against the expected background of $43.16 \pm$ 2.33 (stat.) \pm 5.68 (syst.) events. For the hypothesis of heavy- $W^{\prime}$ production, the respective results are 38 events against the expected background of $41.33 \pm 1.47$ (stat.) \pm 6.88 (syst.) events. We can see that there are no significant deviations from Standard Model predictions. Thus, heavy $Z^{\prime}, W^{\prime}$, and $G$ resonances were not observed in the topologies being considered. New upper limits on the cross sections for the production of these resonances were accordingly established, and the lower limits on their masses were obtained and are presented in Table 2.

\section{SEARCHES OF A HEAVY $t^{\prime}$ QUARK IN EVENTS FEATURING LEPTONS AND JETS}

Searches for fourth generation heavy fermions, whose existence is predicted in many Standard Model extensions, is one of the interesting lines of investigations in searches for new physics. Measurements at the LEP collider made it possible to rule out the existence of fourth-generation neutrinos in the mass range $m\left(\nu_{4}\right)<m_{Z} / 2$, where $m_{Z}$ is the $Z$-boson mass. On the other hand, the mass of such a neutrino cannot be overly large because this would entail the growth of radiative corrections [17]. If we assume that $m\left(\nu_{4}\right) \sim m_{Z} / 2$, then radiative corrections remain small [18], and the existence of heavy quarks having masses around $400 \mathrm{GeV} / c^{2}$ becomes quite possible. It is important to note that, in the models being considered, the admissible mass range 


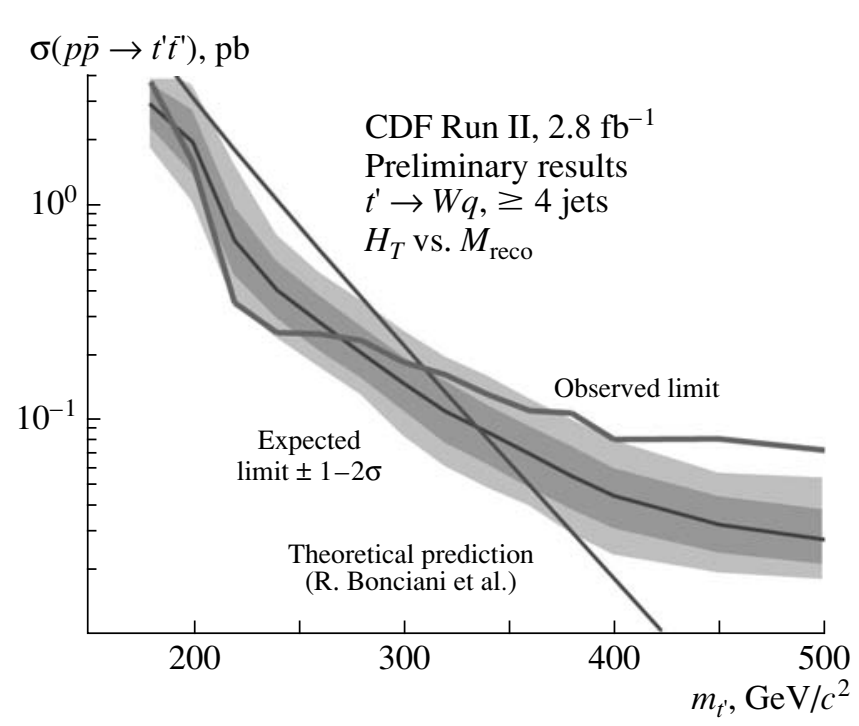

Fig. 8. Limit on the cross section for the double production of a heavy quark $t^{\prime}$ versus its mass along with the theoretical value of the cross section for the double production of $t^{\prime} \bar{t}^{\prime}$.

for Higgs bosons proves to be much wider than in the Standard Model - the upper limit on the Higgs boson mass moves to $500 \mathrm{GeV} / c^{2}$. In such models, the mass difference between heavy $b^{\prime}$ and $t^{\prime}$ quarks proves to be quite modest $\left(m_{b^{\prime}}+m_{W}>m_{t^{\prime}}\right)$; therefore, $t^{\prime} \rightarrow$ $W q$, where $q$ is down-type quark $(d, s, b)$, appears to be a dominant mode of heavy- $t^{\prime}$ decay [19]. Models in which similar heavy quarks arise also include some sypersymmetry models [20] and Little Higgs (LH) models [21].

The CDF Collaboration also presented results of an analysis aimed at searches for the double production of a heavy $t^{\prime}$ quark decaying through the channel $t^{\prime} \rightarrow W q(q=d, s, b)$; events where one of $W$ decayed through a leptonic channel and where the other decayed through a hadronic channel were selected in this analysis. This means that the finalstate signature included a lepton, four jets, and a large missing energy carried away by a neutrino [22]. The total statistical sample used in performing this analysis corresponded to the integrated luminosity of $2.8 \mathrm{fb}^{-1}$.

This analysis did not reveal any significant deviations from Standard Model predictions for masses of the hypothetical $t^{\prime}$ quark in the range extending up to about $400 \mathrm{GeV} / c^{2}$. In the region of higher masses (around $450 \mathrm{GeV} / c^{2}$ ), however, there is some excess of events in the data above the number of events that is predicted on the basis of the Standard Model. Yet, the small number of events in this region gives no way to draw more definitive conclusionsthe available statistics impose stringent limitations on this analysis. In the future, it would be of interest to repeat this analysis for vaster statistics. For the time being, limits on the cross section for the production of a heavy quark $t^{\prime}$ subsequently decaying to $W q$ versus its mass were established (see Fig. 8). The resulting lower limit on the mass of this quark is $311 \mathrm{GeV} / c^{2}$.

\section{CONCLUSIONS}

Although there are many problems inherent in the Standard Model, it works astonishingly well, and the most recent results from experiments at the Tevatron accelerator only confirm this. So far, no data that would provide unambiguous indications of the existence of new physics beyond the Standard Model have been obtained either in the D0 or in the CDF experiment, even though the respective searches have been performed along a wide variety of lines (only some of them are listed in the present article). Yet, it should be noted that the results considered above were obtained on the basis of statistics corresponding to the integrated luminosity of 3 to $4 \mathrm{fb}^{-1}$, but statistics corresponding to an integrated luminosity of about $7 \mathrm{fb}^{-1}$ have been accumulated to date in experiments at the Tevatron; over the time that remains until the completion of accelerator operation, it is planned to accumulate statistics corresponding to an integrated luminosity of 10 to $12 \mathrm{fb}^{-1}$. An increase in statistics by a factor of two to three may prove to be quite sufficient for the discovery of new-physics effects even at the Tevatron. Moreover, the commissioning of the Large Hadron Collider (LHC) will make it possible to increase greatly our potential in this respect.

Much more detailed information about the work already done and the work under way on searches for new physics in the D0 and CDF experiments can be found on the websites of the two experiments [23, 24]. 


\section{ACKNOWLEDGMENTS}

I am grateful to S.P. Denisov, D.S. Denisov, and the headquarters of the New Phenomena Group in the D0 experiment for their help in the preparation of this article for publication.

This work was supported in part by the Russian Foundation for Basic Research (project no. 08-0200607).

\section{REFERENCES}

1. S. Weinberg, Phys. Rev. Lett. 19, 1264 (1967); A. Salam, in Elementary Particle Theory, Ed. by N. Svartholm (Almquist and Wiksells, Stockholm, 1969); S. L. Glashow, J. Iliopoulos, and L. Maiani, Phys. Rev. D 2, 1285 (1970).

2. A. V. Popov, Yad. Fiz. 72, 743 (2009) [Phys. At. Nucl. 72, 702 (2009)]; Yad. Fiz. 73, 1052 (2010) [Phys. At. Nucl. 73, 1015(2010)].

3. D0 Collab., D0 Note 5980 (2009); CDF Collab., CDF Note 9888 (2009).

4. D0 Collab., D0 Note 5935 (2009).

5. CDF Collab., arXiv:0907.1269v3 [hep-ex].

6. V. M. Abazov et al., Phys. Rev. Lett. 103, 061801 (2009).

7. D0 Collab., D0 Note 5937 (2009).
8. T. Sjöstrand et al., Comput. Phys. Commun. 135, 238 (2001).

9. CDF Collab., CDF Note 9834 (2009); CDF Note 9439 (2009).

10. CDF Collab., CDF Note 9817 (2009).

11. D0 Collab., D0 Note 5931 (2009).

12. J. L. Rosner, Phys. Rev. D 35, 2244 (1987).

13. P. Langacker, arXiv:0801.1345 [hep-ph].

14. D0 Collab., D0 Note 5923 (2009).

15. T. Aaltonen et al., Phys. Rev. Lett. 102, 031801 (2009).

16. CDF Collab., CDF Note 9730 (2009).

17. S. Eidelman et al. (Particle Data Group), Phys. Lett. B 592, 1 (2004).

18. G. Kribs et al., ANL-HEP-PR-07-39 (2007); V. A. Novikov, L. B. Okun, A. N. Rozanov, and M. I. Vysotsky, hep-ph/0111028.

19. P. H. Frampton, P. Q. Hung, and M. Sher, Phys. Rep. 330, 263 (2000).

20. H.-J. He, N. Polonsky, and S. Su, hep-ph/0102144.

21. T. Han et al., Phys. Lett. B 563, 191 (2003).

22. CDF Collab., CDF Note 9446 (2008).

23. http://www-d0.fnal.gov/Run2Physics/WWW/results/np.htm

24. http://www-cdf.fnal.gov/physics/exotic/exotic.html Translated by A. Isaakyan 